Luxemburg). Quel est le sens de cette evolution? Evidemment que l'on transforme en prairies des terres de moins en moins propres a cette transformation, mais cependant qui assurent encore un profit aux cultivateurs gráce à la hausse rapide du prix de la viande. C'est encore un aspect de la loi du rendement décroissant.

\title{
Professor Charles Gide (University of Paris).
}

Ma réponse à la question que vous me faites l'honneur de me poser ne sera pas bien longue. Je suis en effet de ceux qui pensent que la hausse actuelle des prix ne peut avoir qu'une cause qui est l'énorme accroissement de la production du métal or. Quoique je n'ignore pas que la theorie quantitative de la monnaie est aujourd'hui démodée, je ne vois pas d'autre cause qui puisse expliquer l'universalité du phénomène dans tous les pays et pour presque tous les articles.

Ceci admis il n'y a évidemment aucun remede à chercher-à moins de limiter la monnayage de l'or et encore serait-il probablement insuffisant. Mais d'ailleurs comme je ne vois aucun mal mais au contraire un bien dans la hausse du prix-un bien pour tous sauf le rentier oisif-je ne vois pas pourquoi on chercherait un remede. Quoique je suis plutôt interventionnale d'opinion, dans ce cas je suis pour le laisser faire.

Néanmoins j'ai approuvé le projet de Conference internationale de Mr. Irving Fisher. Elle pourra rendre des services, non en cherchant de vains remedes à la hausise des prix, mais en l'étudiant au point de vue statistique, en cherchant quels sont les facteurs économiques qui ont co-opéré avec la dépréciation du numéraire ou qui ont agi en sens inverse, et en donnant des indications sur le coat de la vie des classes ouvrietres et sur l'élévation du salaire à laquelle elles auraient droit.

\section{Professor Charles Rist (Professor of Political Economy, University of Montpellier).}

Si j'ai Bien compris l'article de M. Layton, que vous m'avez fait l'honneur de me communiquer, l'auteur considère comme établi que la hausse des prix a fait au total plus de mal que de bien. La question est des plus difficiles a résoudre, plus difficile peut-etre encore que celle des causes de la hausse. Pour la France tout au moins, bien habile serait celui qui pourrait y répondre par un simple oui ou un simple non. On y constate en effet, depuis dix ans, au moins une circonstance tres favorable une grande partie de la communauté, dans notre pays resté relativement plus agricole que nos grands voisins de l'Est et de l'Ouest: c'est la hausse des profits agricoles, qui se traduit par la hausse des fermages et du prix des terres cultiveses, et par une prospérité générale dahs tes campagnes. Cette hausse qui dans un pays de proprieté très concentrée pourrait être considerée comme une privilege échu d une petite minorite-profite au coptraire a une tres large portion de la population dans un pays comme la France ou la propriété et l'exploitation du sol sont encore tres morceltes.

Je sais bien qu'en face de cette circonstance on peut en placer 
d'autres : la baisse du prix des valeurs à revenu fixe (rentes sur l'Etat, obligations et méme actions de chemins de fer)-la hausse du prix des denrées pour le consommateur : ouvriers, fonctionnaires ou personnes appartenant aux professions liberales! Mais d'abord la baisse du prix des valeurs à revenu fixe est compensée par une hausse considérable de l'intérêt et des profits industriels; et, malgré la légende soigneusement entretenue d'une vaste démocratie financière française - (qui n'en est pas moins une simple légende)-cette baisse a frappé dans l'ensemble des personnes parfaitement à même de la compenser par de profitables placements.

Pour les consommateurs la question est plus complexe. Cependant dans un pays de fortunes relativement divisées, et où (à part la classe ouvrière) chaque individu participe à des sources très diverses de revenu, il y a eu sans doute compensation dans une foule de cas. Je sais bien que partout l'on se plaint de la vie chère, mais vous savez comme moi qu'en matière économique on se plaint très fort de ce qu'on perd, - et l'on est très discret sur ce qu'on gagne. Aussi je crois qu'au total l'impulsion nouvelle donnée a l'activité économique par la hausse des prix est le fait dominant.

Reste la classe ouvrière. C'est le point noir. Jusqu'en 1910 d'après une enquête de $M$. March, l'eminent dírecteur de la Statistique de la France, son salaire réel avait continué à progresser. Mais depuis? j'ai bien peur qu'ici, la hausse énorme de ces deux dernières années n'ait eu des conséquences néfastes. Faut-il attribuer en partie à la hausse des prix l'augmentation du nombre des grèves? nombre qui a passé en moyenne de 727 par an pendant la période $1900-1905$, à 1277 par an pendant la période 1906-1911? C'est très possible. D'autant plus qu'il $y$ a là un phénomène général en Europe-où l'on constate pour l'Angleterre, l'Allemagne, la Belgique, l'Italie et l'Autriche prises ensemble, une augmentation de $54 \%$ d'une période à l'autre, dans le nombre annuel des grèves.

J'en conclus qu'il sera très difficile-devant l'opposition des intérêts en cause-de persuader les gouvernements de la bienfaisance des mesures préconisées pour parer la la hausse des prix. D'autant plus que l'histoire politique (celle du continent, sinon celle de l'Angleterre) nous prouve qu'il est beaucoup plus aisé de convaincre un gouvernement d'agir en faveur d'une hausse des prix qu'en faveur d'une baisse. Aussi je souhaite que la commission internationale qui se réunira, se borne à examiner le problème purement scientifique et déja très délicat des causes et des effets de la hausse, et qu'elle relègue au second plan les questions pratiques. Ne sortirait-il de travaux que la démonstration de ce fait: que la plupart des grands problemes économiques sont devenus internationaux, et doivent se résoudre internationalement qu'il faudrait déjà saluer avec reconnaisance l'initiative si heureusement prise par MM. Bauer et Fisher.

\section{Professor S. J. Chapman (University of Manchester).}

Mr. Layton's paper draws attention to one of the most serious economic movements at the present time. Variations in general prices always result in grave consequences, and it is not easy to say offhand whether rising or falling prices (when the rise or fall is 